\title{
A Wearable Device For Physical and Emotional Health Monitoring
}

\author{
Srinivasan Murali ${ }^{1}$, Francisco Rincon $^{1,2}$, David Atienza ${ }^{2}$ \\ ${ }^{1}$ SmartCardia, Lausanne, Switzerland \\ ${ }^{2}$ Embedded Systems Laboratory (ESL), EPFL, Lausanne, Switzerland
}

\begin{abstract}
Personal health monitoring systems are emerging as promising solutions to develop ultra-small, portable devices that can continuously monitor and process several vital body parameters. In this work, we present a wearable device for physical and emotional health monitoring. The device obtains user's key physiological signals: ECG, respiration, Impedance Cardiogram (ICG), blood pressure and skin conductance and derives the user's emotion states as well. We have developed embedded algorithms that process the bio-signals in real-time to detect any abnormalities (cardiac arrhythmias and morphology changes) in the ECG and to detect key parameters (such as the Pre-Ejection Period and fluid status level) from the ICG. We present a novel method to detect continuous beat-by-beat blood pressure from the ECG and ICG signals, as well as a realtime embedded emotion classifier that computes the emotion levels of the user. Emotions are classified according to their attractiveness (positive valence) or their averseness (negative valence) in the horizontal valence dimension. The excitement level induced by the emotions is represented by high to low positions in the vertical arousal dimension of the valence-arousal space. The signals are measured either intermittently by touching the metal electrodes on the device (for point-of-care testing) or continuously, using a chest strap for long term monitoring. The processed data from device is sent to a mobile phone using a Bluetooth Low Energy protocol. Our results show that the device can monitor the signals continuously, providing accurate detection of the motion state, for over 72 hours on a single battery charge.
\end{abstract}

\section{Introduction}

With the advent of smart phones, continuous health care delivery has become easier. Today, there are several vital parameter-sensing platforms that use the mobile phone as a gateway (and display), and have the capability to directly alert the doctor or hospital in case of a problem with the health condition [1]. There is a recent explosion of wearable devices, such as the Apple Watch [2] and Jawbone
UP3 [3], which are collecting a plethora of continuous vital signs, providing an opportunity to identify and prevent non-communicable diseases effectively. Autonomous systems that can provide quick and continuous health status monitoring of people are surely desirable and can improve personal health as well as lower health costs for society. Such systems can monitor the vital parameters, such as the electrocardiogram (ECG), pulse and oxygen saturation, blood pressure, at home as well in outdoor environments, keep the user informed about his health conditions and/ or, alert medical doctors in case of emergencies.

While continuous mobile health monitoring opens up new healthcare delivery mechanisms, it also creates new challenges. One of the most important challenges is to obtain relevant and meaningful health information from the continuous stream of vital signs, with the acquisition of the relevant signals from a single point-of-care device.

In this work, we present a multi-parametric device that acquires important vital signs. It has a small, light-weight monitor and a special purpose computer chip inside that can continuously record, process and store the ECG/EKG waveform, ICG, skin conductance and respiration of a person. The monitor consists of four non-invasive electrode sensors attached to the skin of a patient, which are linked to a radio module. The device can be used by a simple touch, where the user touches the four pads, or worn continuously on the chest using a chest strap. It streams the data and results to the patients mobile phone or a hub node and then to the doctor/hospital center.

We have developed algorithms that run on the device to perform the following tasks:

- Isolate the different signals, as well as filter and analyze the individual signals.

- Remove muscle noise and compensate for movementinduced noise of users and power supply noise.

- Extract significant features of the signal, and delineate the different waveform patterns. This information can be used to detect anomalous waveform patterns and detect different arrhythmia conditions.

- On-device machine learning, to perform data fusion of the feature sets extracted from the different signals and combine them to estimate physical and emotional health. 


\section{Previous Work}

Wireless Body Sensor Nodes (WBSNs) are embedded sensor platforms for bio-signal acquisition. They offer a compelling solution for long-term monitoring of patients, presenting little discomfort for the subjects and requiring minimal supervision from medical staff. Proposed WBSNs are able to acquire and wirelessly transmit different bio-signals (e.g.: blood pressure, pulse oximetry and electrocardiograms [4]). In addition to acquisition and wireless transmission of bio-signals, state-of-the-art WBSNs embed advanced processing applications, able to automatically retrieve diagnostic information from the acquired data [5]. By employing on-node processing, only the analysis results (as opposed to acquired data) has to be transmitted on the power-hungry wireless link, substantially increasing the energy efficiency [6], which is a crucial factor for WBSNs.
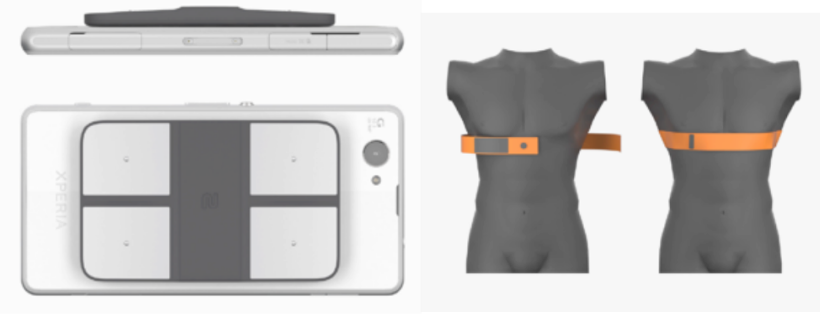

Figure 1. Device for physiological signal acquisition. Device can be attached behind a phone, and the metal pads touched by the user or can be attached using a chest strap.

\section{Signal Acquisition Platform}

The signal acquisition hardware developed is shown in Figure 1. Two of the metal pads are used to sense the ECG of the user. In two of the pads (one on each side), a minute current is injected and the ICG is sensed from the other two pads. The ICG signal is modulated by the respiration of the user, and that is obtained as well. The skin conductance is obtained from the two pads on the same side. As all the different signals are obtained from the same set of pads, the signals are super-imposed on each other and are extracted individually using embedded processing methods. In the rest of this section, we present the different signal acquisition and processing in detail.

\subsection{Impedance Cardiogram}

ICG is a technique used for hemodynamic parameter measurement (such as thoracic fluid level, stroke volume and cardiac output) [7]. In traditional ICG, four to six electrodes are placed across the thorax, with a high frequency, low amplitude current injected across the outer electrodes and the voltage drop (and hence the impedance) measured from the inner electrodes. ICG measures the variations in impedance caused by the change in the blood volume and velocity at the aorta with each heart beat. Thus, ICG provides information about the heart muscle activity, and a complementary signal to the ECG. Several clinical studies have shown that the ICG can provide a marker for the ejection fraction of Echocardiogram [8]. In our device, we inject a low amplitude current (below $10 \mu \mathrm{A}$ ) at different frequencies (between $1 \mathrm{KHz}$ to $1 \mathrm{MHz}$ ) across the metal pads. The custom designed front-end obtains the voltage drop across the other two metal electrodes. The metal pads can be placed on the chest, or can also be touched by fingers to obtain the impedance changes. The ICG signal is sampled at $250 \mathrm{~Hz}$, with 16-bit resolution.

\subsection{ElectroCardiogram}

A single lead ECG is obtained across two of the metal pads that are touched by different hands. The ECG is sampled at a configurable rate, from $250 \mathrm{~Hz}$ to $1 \mathrm{KHz}$ (for pacemaker pulse detection). The ECG signal is separated from the ICG and processed on device to remove muscular noise. Several different noise filtering methods have been implemented in our earlier work [6]. The signal is further processed to delineate the different $\mathrm{P}, \mathrm{QRS}$ and $\mathrm{T}$ waveforms. Cardiac arrhythmias and morphological changes can be detected from the delineated signal

\subsection{True Respiration}

The impedance measurements are not only affected by the blood volume changes with heart beat, but also by several other factors, such as the body fluid and composition levels, posture and breathing. While the base value of impedance is affected by the composition level and posture, breathing induces a shift in the waveform correlating with the breathing pattern. By removing the higher frequency oscillations (of the heart-rate), the lower frequency of breathing rate is obtained.

\subsection{Skin Conductance}

Skin conductance level and response (latter also called as Galvanic Skin Response) reflect the variation of skin resistance with the state of the sweat glands. The sympathetic nervous system modulates the sweat glands, with increased arousal resulting in larger responses of sweat glands and hence, the skin conductance. It's an important signal used for emotion analysis. In our device, we obtain the skin conductance across two pads that are touched by the same hand. Skin conductance measurement is done at $100 \mathrm{~Hz}$. 

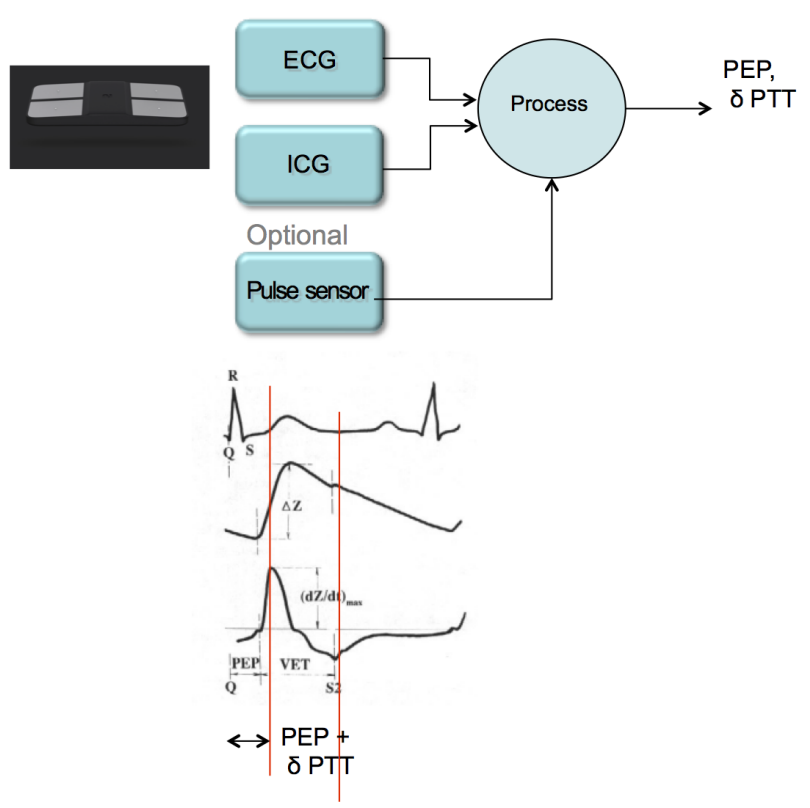

Figure 2. Estimation of NIBP using ECG, ICG and optionally a pulse sensor. The bottom part of the figure shows the ECG, ICG and derivative of ICG waveforms. The PEP and $\delta$ PTT are obtained from the ECG and ICG signals.

\section{Non-Invasive Blood Pressure Estima- tion}

Several works have addressed the issue of measuring Non-Invasive Blood Pressure (NIBP) without requiring a pressure cuff [9]. One of the most promising approaches for continuous NIBP measurement is the use of Pulse Transit Time (PTT): the time it takes for the blood to flow from aorta to a peripheral part of the body, such as the fingers. Several works estimate the NIBP by detecting timing information from ECG and pulse signal (using an optical sensor) obtained at the finger. However, such a measurement is not accurate to track diastolic pressure, as the ECG doesn't reflect the timing information on when the aortic valve opens [9]. A more accurate method is to use the ICG signal as well, as characteristic points in the ICG waveform reflect the opening of the aortic valve. By measuring the signal at the fingers, we capture a combination of both aortic valve opening and the peripheral blood flow on the ICG waveform. Combining the ECG and ICG, we can obtain the $\delta$ PTT (a portion of PTT), as well as the Pre-ejection Period (PEP), which is the timing difference between the R-peak of ECG waveform and the aortic valve opening that is observed in ICG. This is shown in Figure 2.

\section{Emotion Detection}

Analysis of human emotions has several different applications in building affective computing systems [10]: monitoring of patients with disorders, such as epileptic seizures or ADHD, to monitoring stress levels of workers and automobile drivers [11]. Different approaches, such as gesture recognition, speech and text analysis and analysis of the autonomic nervous system (ANS) have been used for emotion analysis [10]. Several research works have shown the effects of emotions on the ANS that can be observed in the physiological signals [13].

Common physiological signals that are used for emotion analysis from the ANS include the ECG, ElectroMyoGram (EMG), respiration and skin conductance. Emotion analysis is typically performed on a valence-arousal model [13], wherein the valence scale is used to represent positive to negative emotions and the arousal scale is used to represent the excitement/relaxation level. Thus, a high valencehigh arousal state would be joy, high valence-low arousal state would be happy, low valence-high arousal state would be anger and low valence-low arousal state would be sad. A better granularity of classification can lead to identifying further emotions as well.

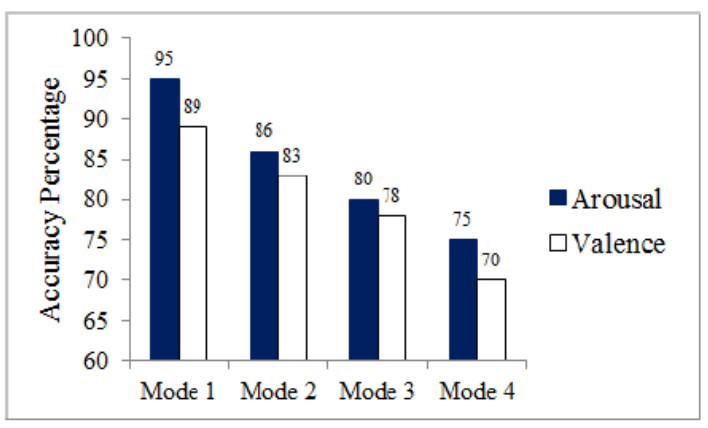

Figure 3. Accuracy of detection of the different ValenceArousal states in different operating modes.

\begin{tabular}{c|c|c} 
Mode & Average current $(\mathrm{mA})$ & Lifetime $(\mathrm{h})$ \\
\hline 1 & 4.86 & 146.1 \\
\hline 2 & 1.69 & 420.1 \\
\hline 3 & 1.09 & 651.3 \\
\hline 4 & 0.63 & 1126.9 \\
\hline
\end{tabular}

Figure 4. Current consumption and battery life time of the system under the different modes

As obtaining an ultra-low power operation is crucial for long term monitoring using the wearable device, we have developed a mode-based emotion analysis engine. Based on the application targeted, the accuracy required for emo- 
tion detection can vary. For example, in order to detect the arousal levels during epileptic seizure, a higher accuracy of emotion classification is desirable, while for an application such as gaming, a lower accuracy is acceptable. We have developed a system where in 4 different energy-accuracy modes can be used for emotion detection. The details of the classifier design and feature selection is presented in detail in our earlier work [14]. The accuracy of detection of valence-arousal modes and the energy requirements (and hence the battery life of the system) on test data sets are shown in Figures 3 and 4. For the experiments, we use the Augsburg dataset [15]. The dataset has recordings of four emotions (anger, joy, sadness and pleasure) over 20 days, thereby having 80 recordings in total and tagged with the ground truth from the user. We trained the classifier using a part of the data and tested the classification accuracies across the rest of the data set.

\section{Conclusions}

In this paper, we presented a wearable device for physical and emotional health monitoring. The device obtains multiple vital signs simultaneously. Signal filtering and separation is done on device, with important features of different signals extracted. The multiple features are combined to obtain insights about the physical health (such as the blood pressure variations) and emotional health of the users. In future, we plan to develop adaptive classifiers to customise the learning patterns to individual users.

\section{Acknowledgements}

This work has been partially supported by the BodyPoweredSenSE (no. 20NA21 143069) RTD project, evaluated by the Swiss NSF and funded by Nano-Tera.ch with Swiss Confederation financing, as well as the NCCR Robotics - Sub-project 9.2: Bidirectional smart jacket.

\section{References}

[1] E. Jovanov, et al. , "Patient Monitoring Using Personal Area Networks of Wireless Intelligent Sensors," Biomedical Sciences Instrumentation, vol. 37, pp. 373-378 2001.

[2] Apple Watch. Online: https://www.apple.com/watch/

[3] Jawbone UP. Online: https://jawbone.com/up/

[4] J. Parkka et al. 'Activity classification using realistic data from wearable sensors'. In: IEEE Transactions on Information Technology in Biomedicine 10.1 (2006), pp. 119-128. issn: 1089-7771.

[5] Y. Hao and R. Foster. 'Wireless body sensor networks for health-monitoring applications'. In: Physiological Measurement 29.11 (Oct. 2008), R27.

[6] F. Rincon et al. 'Development and Evaluation of Multilead Wavelet-Based ECG Delineation Algorithms for Embedded Wireless Sensor Nodes'. In: Information Technology in Biomedicine, IEEE Transactions on 15.6 (Nov. 2011), pp. 854-863.

[7] G. H. M. Willemsen et al., "Ambulatory monitoring of the impedance cardiogram", Psychophysiology, 33: 184193, 1996.

[8] CW Parott et al., "Comparison of Changes in Ejection Fraction to Changes in Impedance Cardiography Cardiac Index and Systolic Time Ratio", Congest Heart Fail. 10 (Suppl 2) 2004:11-13.

[9] J. M. Sola, Continuous non-invasive blood pressure estimation, Ph.D. dissertation, ETH Zurich, 2011.

[10] R. Picard, Affective computing, MIT Technical Report, 1995

[11] M.-Z. Poh et al., Continuous monitoring of electrodermal activity during epileptic seizures using a wearable sensor, in Engineering in Medicine and Biology Society (EMBC), 2010 Annual International Conference of the IEEE, Sept. 2010, pp. 44154418.

[12] S. Kreibig, Autonomic nervous system activity in emotion: A review, Biological Psychology, vol. 84, no. 3, pp. 394 421, July 2010.

[13] S. Koelstra et al., Deap: A database for emotion analysis ; using physiological signals, IEEE Transactions on Affective Computing, vol. 3, no. 1, pp. 18 31, June 2011.

[14] M. Padmanabhan et al., "Energy-Aware Embedded Classifier Design for Real-Time Emotion Analysis", EMBC 2015.

[15] J. Wagner et al., From physiological signals to emotions: Implementing and comparing selected methods for feature extraction and classification, in IEEE Intl. Conf. Multimedia and Expo (ICME), 2005, pp. 940943.

Address for correspondence:

Srinivasan Murali

EPFL Innovation Park, Batiment C, CH1015, Switzerland.

srinivasan.murali@smartcardia.com 